\title{
e-Phaïstos
}

e-Phaïstos

Revue d'histoire des techniques / Journal of the history

of technology

V-2 | 2016

Histoire des techniques en Afrique de l'Ouest

\section{La rencontre entre patrimoine matériel et immatériel dans l'architecture du Soudan central : études de cas}

The Meeting between the Tangible and Intangible Heritage in the Architecture

of Central Sudan: Case Studies

\section{Addo Mahamane}

\section{OpenEdition}

Journals

Édition électronique

URL : http://journals.openedition.org/ephaistos/5779

DOI : 10.4000/ephaistos.5779

ISSN : 2552-0741

Éditeur

IHMC - Institut d'histoire moderne et contemporaine (UMR 8066)

Édition imprimée

Date de publication : 15 juin 2016

Pagination : 49-63

ISSN : 2262-7340

Référence électronique

Addo Mahamane, «La rencontre entre patrimoine matériel et immatériel dans l'architecture du Soudan central : études de cas », e-Phaïstos [En ligne], V-2 | 2016, mis en ligne le 15 mars 2020, consulté le 17 juin 2020. URL : http://journals.openedition.org/ephaistos/5779 ; DOI : https://doi.org/ 10.4000/ephaistos.5779 


\title{
La rencontre entre patrimoine matériel et immatériel dans I'architecture du Soudan central : étude de cas $^{1}$
}

\author{
Addo Mahamane \\ Maître de Conférences \\ Université Abdou Moumouni \\ Faculté des Lettres et Sciences \\ Humaines \\ Département d'Histoire. \\ Recteur de I'Université de Tahoua
}

\section{Introduction}

Le Soudan central et occidental présente un style original d'architecture, au point où l'on peut parler de style régional d'architecture, à travers les palais royaux $^{2}$, les mosquées 3 et les autres monuments historiques 4 . Très peu d'études ont été consacrées à cet important patrimoine architectural, même si certaines de ses composantes ont été classées patrimoine de l'humanité par l'UNESCO5. Ces nombreux monuments, souvent très anciens, témoignent du niveau de technicité atteint par les habitants de la sous-région soudano-sahélienne ${ }^{6}$. L'article se propose de présenter le matériau avant de l'analyser, l'interpréter et dégager les points de convergence et de divergence entre le patrimoine matériel et immatériel de l'architecture soudanienne du Sahel, à partir d'études de cas comme la mosquée d'Agadez, la tour de Gobarau, l'immeuble de Dodo, la mosquée de Tombouctou. L'objectif de cet article est de mettre en évidence les spécificités de l'architecture de l'Afrique précoloniale au sud du Sahara, qui présente une certaine constance depuis l'Égypte ancienne jusqu'à nos jours. Les matériaux utilisés dans cette architecture, depuis les pyramides d'Égypte jusqu'à la tour de Gobarau en passant par les autres cas que nous traitons ici (le mur de Zimbabwe, les ruines de Loropeni au Burkina $\mathrm{Faso}^{7}$ ) relèvent d'un savoirfaire technique particulier. Ce sont des bâtiments ne comportant ni fer ni ciment, résistant au temps, tandis que des bâtiments construits précisément avec du ciment et du fer et considérés comme pérennes s'écroulent chaque année dans la sousrégion ${ }^{8}$. Les techniques utilisées dans cette architecture historique méritent donc une attention particulière des chercheurs et des constructeurs puisqu'elles pourraient apporter des solutions aux problèmes rencontrés à l'heure actuelle. Ce vaste et ambitieux projet ne peut pas être traité exhaustivement dans le cadre d'un seul article, mais nous allons néanmoins en poser les fondements et dégager des pistes de recherche.

\section{Les matériaux d'analyse}

De nombreuses mosquées semblent avoir été construites sous l'égide de Mansa Moussa ${ }^{9}$. Ainsi, selon plusieurs historiens ${ }^{10}$, il ramena au Mali à la suite de son célèbre pèlerinage

« le poète d'origine espagnole, Abou Ishaq dit Es Sahéli ${ }^{11}$ [...] qui construisit des mosquées à Tombouctou, à Gao, à Diré, à Goundam, à 
Ouanka, ou encore à Bako. Des mosquées-écoles furent édifiées dans toutes les agglomérations importantes[...]. Les grands dignitaires politiques et religieux en firent autant et l'architecture s'épanouit avec un cachet nouveau, le style soudanais... ${ }^{12}$.

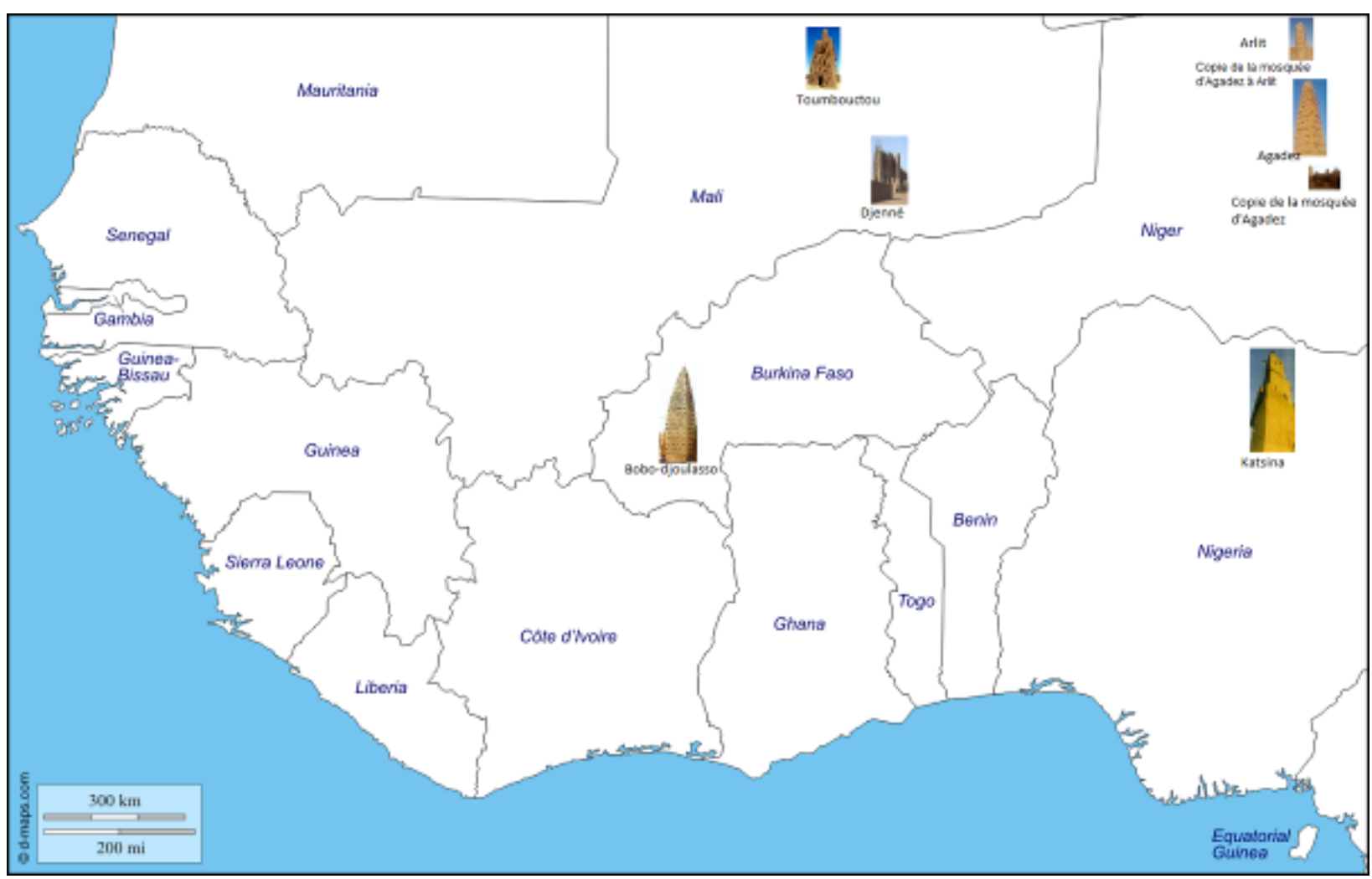

\section{LEGENDE}

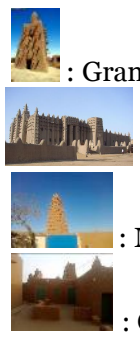

Grande mosquée de Tombouctou

: Grande mosque de Djenné

: Mosquée d’Agadez, au Niger

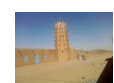

: Copie de la mosquée d'Agadez à Arlit

: Copie de la mosquée d'Agadez à Agadez

Figure 1 : Localisation géographique des échantillons examinés en Afrique de l'Ouest. Source : http://www.histgeo.acaix-marseille.fr/webphp/carte.php?num car=.31.3\&lang=fr (consulté le 26/o4/2016) 
Toutes classées sur la liste du Patrimoine mondial de l'Unesco depuis 1988, les trois grandes mosquées de Tombouctou, comptant parmi les plus anciennes mosquées monumentales de l'Afrique occidentale encore utilisées, sont celles de Djingareyber, de Sankoré et de Sidi Yahya (ou Sidi Yahiya). La plus célèbre et la plus imposante, la mosquée de Djingareyber, a été construite vers 1325. L'empereur Mansa Moussa gratifia Abou Ishaq es-Sahéli de 40000 mitqals $(200 \mathrm{~kg}$ ) d'or pour son érection. Plus grande mosquée de Tombouctou, elle peut accueillir la prière du vendredi qui rassemble jusqu'à $12000^{13}$ personnes.

\section{La grande mosquée de Tombouctou (Mali)}

Elle a été réalisée entièrement en banco $^{14}$, dans le style architectural soudanais. Elle est constituée de vingtcinq files de piliers ${ }^{15}$. Le nombre important de piliers, nécessaires pour limiter la portée des voûtes, ne permet pas d'avoir une vue d'ensemble de la salle. Une fois par an, la population participe à la réfection des enduits extérieurs. Elle est inscrite sur la liste du patrimoine mondial en péril depuis 1989, et bénéficie depuis 1996 d'un financement du Fonds du patrimoine mondial de l'UNESCO pour un projet intitulé «Sauvegarde des mosquées de Tombouctou $»^{16}$.

\section{La grande mosquée de Djenné (Mali)}

La Grande mosquée de Djenné ${ }^{17}$ est considérée par de nombreux

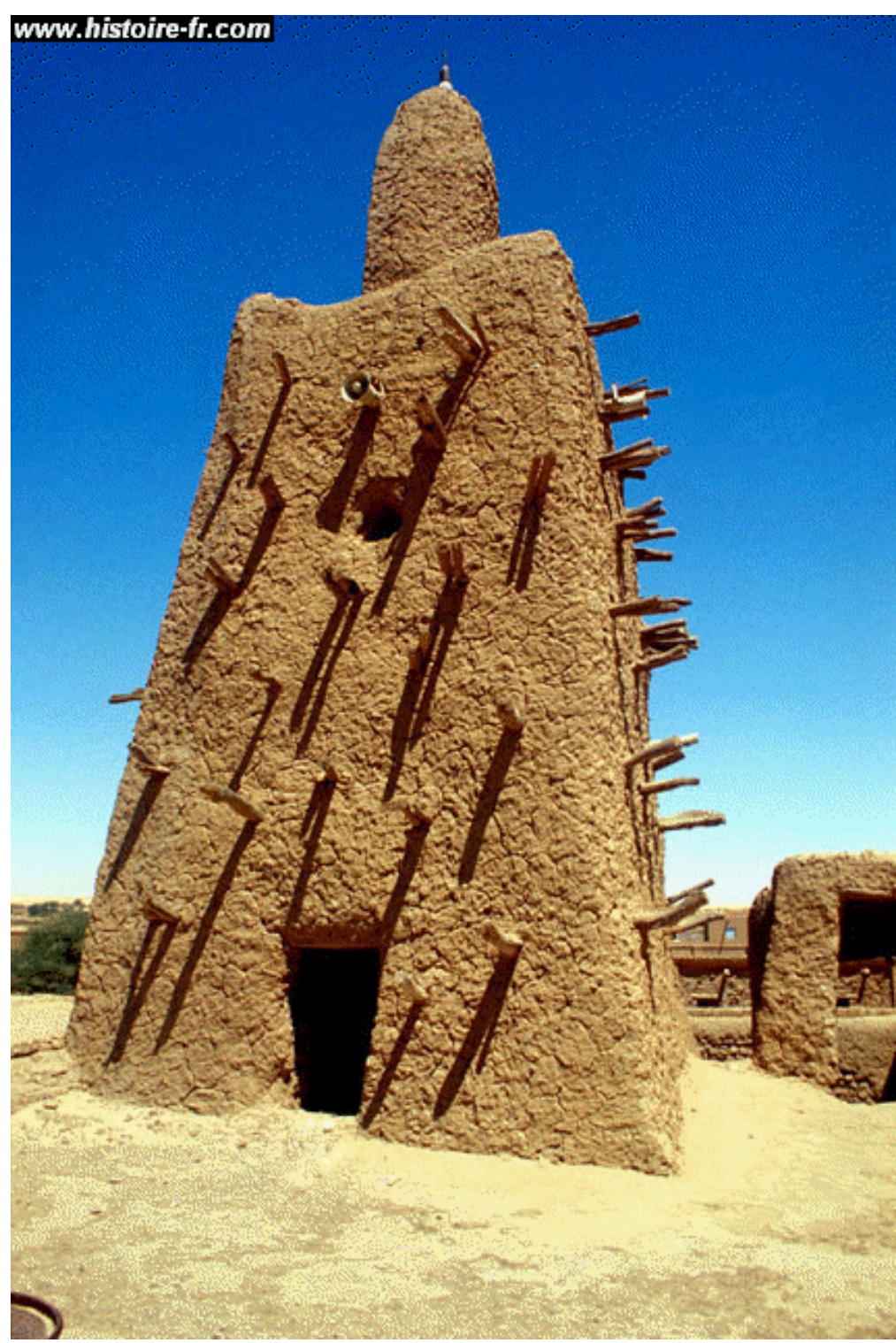

Figure 2 : La grande mosquée de Tombouctou ou Djingareyber, au Mali ; Source : http://yacinfo.over-blog.net/article-reseaux-en-classe-introduction-au-reseau-3826070o.html consulté le 26 octobre 2013 


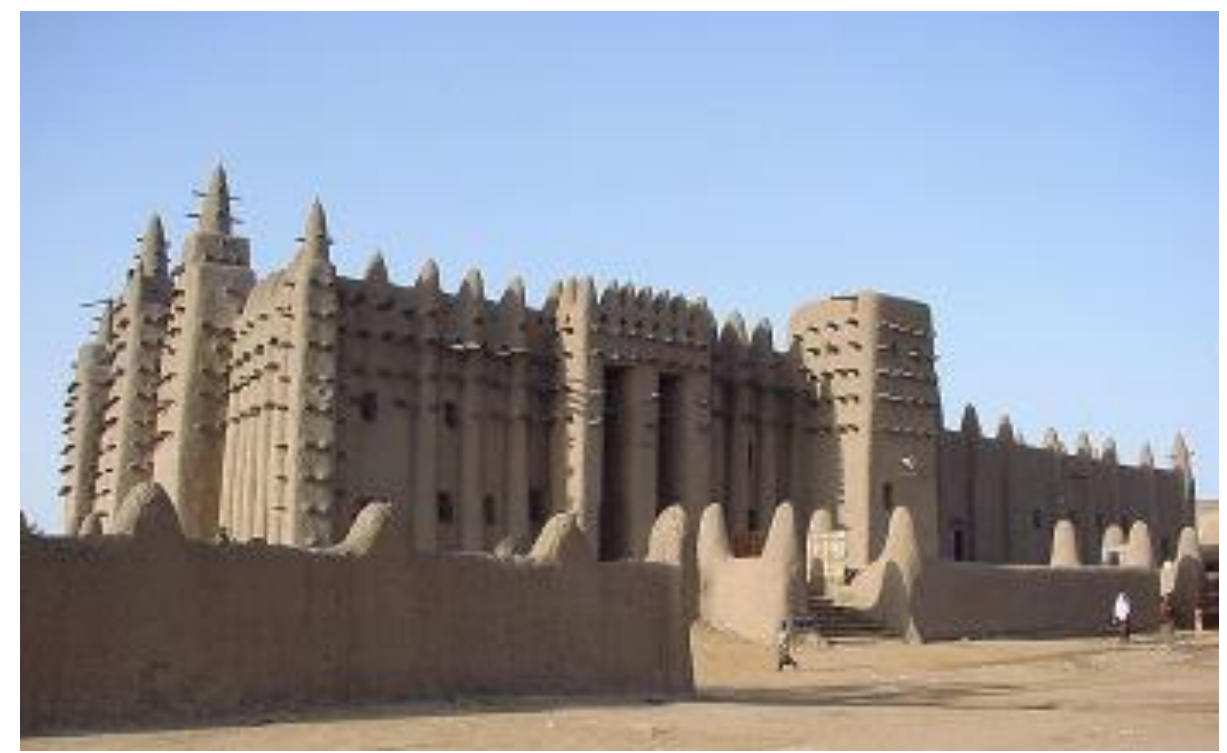

Figure 3 : La mosque de Djenné, au Mali ; Source : wikipedia.org, consulté le 16/o9/2008

architectes et chercheurs comme la réalisation majeure du style architectural soudano-sahélien, tout en reflétant des influences islamiques. La mosquée est située dans la cité de Djenné, au Mali, dans la plaine alluviale du Bani, affluent du Niger. Un premier édifice aurait été construit en ce lieu au XIII siècle, mais la construction actuelle date seulement des environs de 1907. Marquant le centre de l'agglomération de Djenné, c'est aussi l'un des monuments les plus emblématiques de l'Afrique subsaharienne. De concert avec la ville de Djenné elle-même, elle est inscrite depuis 1988 sur la liste du patrimoine mondial de l'UNESCO.

La mosquée a la forme d'un carré de 75 mètres de côté. Sa hauteur est de 20 mètres. Le toit est soutenu par 90 piliers et possède 104 trous d'aération. Elle peut contenir mille personnes. Le site de la Grande mosquée de Djenné était précédemment occupé par un palais. Des experts en architecture islamique tels que Labelle Prussin pensent que ces constructions coniques furent intégrées dans la conception des mosquées dans tout le Mali, et considèrent que la Grande mosquée de Djenné en est l'exemple le plus révélateur.

Ce site a été occupé par des mosquées depuis la construction qui fut ordonnée en 1240 par Koi Kunboro, avant que Djenné ne devienne l'une des principales villes des empires du Mali puis du Songhai. Le conquérant peul Amadou Lobbo, qui enleva Djenné à la suite d'une guerre sainte, fit démolir cette mosquée. Il estimait que le bâtiment d'origine, un palais transformé, était trop riche. La seule partie de ce bâtiment qui a été conservée est un enclos contenant les tombes de chefs locaux. Une restitution de l'édifice, à l'identique, fut terminée en 1896, mais fut ensuite démolie à nouveau pour faire place au bâtiment actuel alors que Djenné relevait du territoire de l'Afrique Occidentale française.

En 1906, le gouverneur colonial français William Ponty acceptait, à la demande du marabout 
Almamy Sonfo, de reconstruire à l'identique l'ancienne mosquée du roi Koi Kunboro.

Achevée en 1907, cette construction fut supervisée et dirigée par le chef de la corporation des maçons de Djenné, Ismaïla Traoré. À l'époque, l'autorité française aida administrativement et économiquement à l'édification de la mosquée ainsi que d'une madrassa voisine.

\section{La tour de Gobarau au Katsina (Nigéria)}

Hasumiya Gobarau (tour de Gobarau), ou mosquée de Gobarau ${ }^{18}$, est l'un des rares monuments historiques de l'aire culturelle katsina. L'historien du pays hausa en général et du katsina en particulier est confronté à une difficulté : les sources écrites sont rares ou tardives, mais les récits oraux populaires locaux sont riches. L'histoire de la tour de Gobarau ne fait pas exception à la règle.

La tour de Gobarau (ou Gobirau ou Goborau selon les prononciations) est un bâtiment de 50 pieds situé dans le centre de la ville de Katsina, au nord du Nigéria. Il est reconnu comme exemple précoce de l'architecture musulmane, dans une ville réputée être un centre théologique renommé. Ce bâtiment est désormais l'un des symboles de la ville et marque ainsi son identité. Gobarau est réputé être, dans les traditions katsina, le premier bâtiment de plusieurs étages en Afrique de l'Ouest. Il est supposé avoir été construit au XVe siècle, pendant le règne $\mathrm{du}$ premier roi du royaume $\mathrm{du}$ Katsina (Sarkin Katsina) Muhammadu Korau (1445-1493/4). D'autres sources ${ }^{19}$ situent sa première construction entre le XVIe et le XVIII

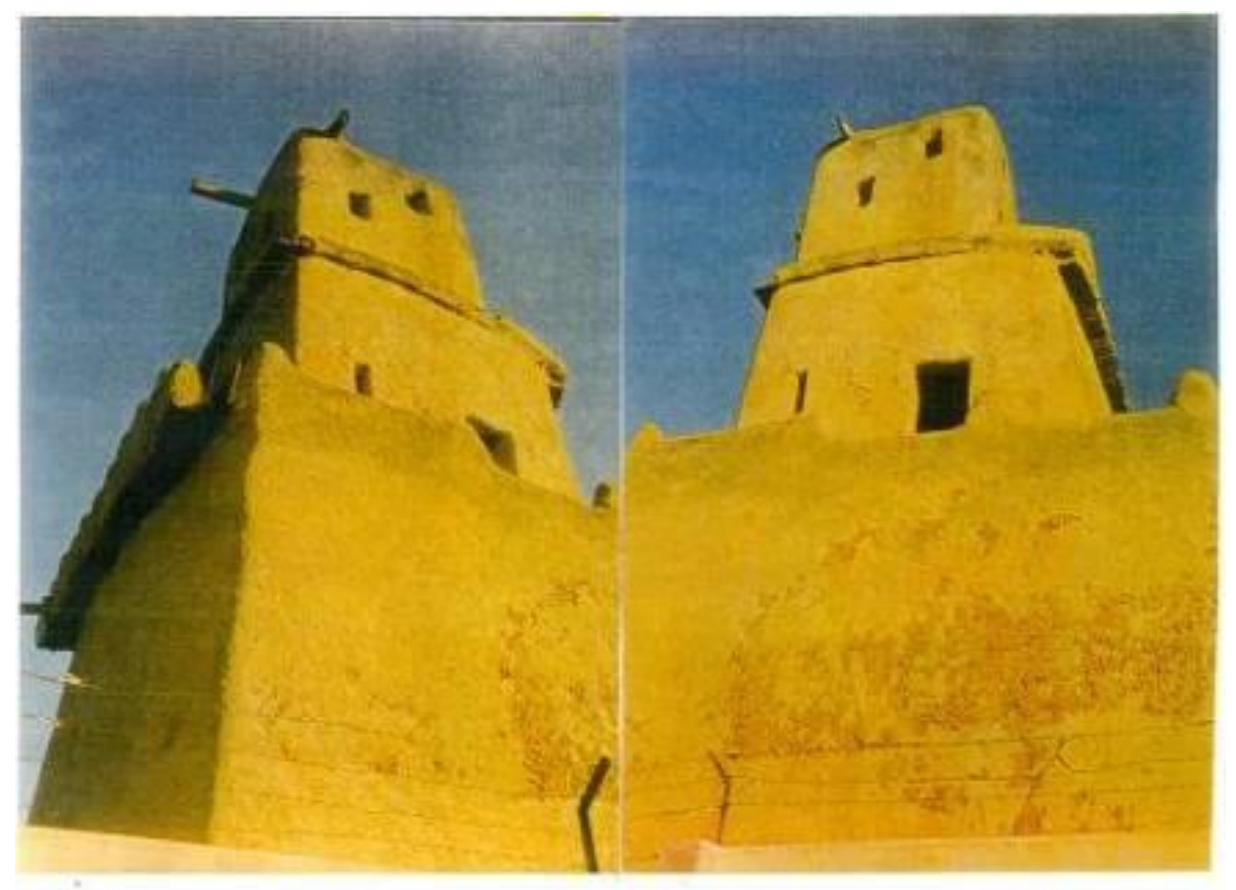

Figure 4 : La tour Gobarau à Katsina, au Nigéria. Source : photographie de A. Mahamane, Birnin Katsina, le 7 mai 1996 
siècles, avant d'être reconstruit au $\mathrm{XX}^{\mathrm{e}}$ siècle. Construit à l'origine comme la mosquée centrale de la ville de Katsina, il a ensuite été utilisé également comme une école. Au début du XVIe siècle, Katsina était devenue un centre commercial et académique très important dans le pays hausa (Kasar Hausa) et Gobarau était devenue une institution célèbre de l'enseignement supérieur islamique. Gobarau a continué à être la mosquée centrale de Katsina jusqu'au début du XIX ${ }^{\mathrm{e}}$ siècle, lorsque Sarkin Katsina Ummarun Dallaji (1805-1835) construisit une nouvelle mosquée, qui fut ensuite démolie par Muhammad Dikko (1906-1944), qui fit ériger le célèbre Masallacin Dutsi, encore utilisé aujourd'hui. La mosquée et la tour ont été rénovées par Sarkin Katsina Muhammadu Kabir Usman (1981-2008).

Le minaret de Gobarau est considéré comme l'un des plus hauts bâtiments de l'État de Katsina. L'histoire de cette tour est liée à celle de l'islamisation du pays selon l'histoire orale ${ }^{20}$. Ainsi, la tour est réputée avoir été construite il y a 664 ans (v.1350). À l'origine, la hauteur du minaret aurait été de plus de $122 \mathrm{~m}$ (mais ce chiffre n'est pas vérifié) avant qu'il ne soit réduit à sa hauteur actuelle de 17.11 mètres environ, avec une base d'environ 83,36 mètres carrés. La tour s'effondra en raison de négligences et du manque d'entretien avant d'être reconstruite par l'émir Muhammad Dikko ${ }^{21}$. Nous devons à la Chronique de Kano les premières précisions sur les différentes étapes de l'islamisation du Katsina. Selon cette chronique, les sarakunan Katsina étaient des anna (annimistes) jusqu'à la fin du règne de Jibda Yaki (v.1405-1445). Le sarkin Katsina Muhammad Korau (14451493/4) aurait été le premier sarki à avoir tenté de faire de l'islam une religion d'État au Katsina. Cette œuvre religieuse de Korau fut poursuivie par son successeur immédiat Ibrahim Sura (v. 1493/41495/6). La Chronique de Kano précise qu'Ibrahim Sura commença la construction de la ville de Katsina. Puis, elle précise les œuvres d'islamisation d'un autre sarkin Katsina, Maje Ibrahim. Jusqu'au règne de Maje Ibrahim, la Chronique de Kano ne mentionne pas l'Hasumiya Gobarau. Il est certain qu'à la fin du XVe siècle, la réputation islamique de kasar Katsina est établie. Rappelons que c'est sous le règne d'Ibrahim Sura que l'Égyptien al-Suyuti (1445-1495) adressa une lettre à certains souverains du Soudan central, dont Ibrahim Sura, pour qu'ils dirigent leur pays selon les recommandations de l'islam. Mais cette lettre ne parle pas non plus de la tour de Gobarau. Malgré le jihad de 1804, au XIX siècle on ne dispose pas de témoignages écrits sur Hasumiyar Gobarau. Les sources écrites mettent en revanche l'accent sur la réputation du Katsina comme un pays comportant des centres islamiques et des marabouts célèbres ${ }^{22}$. Mais ces sources ne permettent pas de situer dans le temps la construction de Hasumiyar Gobarau.

Selon certaines sources, Hasumiyar Gobarau n'est pas aussi ancienne que le dit la tradition orale ${ }^{23}$ puisqu'elle aurait été construite par Sarkin Katsina Gozo (1796-180124). La tour fait partie, avec les saints Dan Masani, Dan Marina, ou encore Dan Takum, des héritages qui déterminent l'identité de birnin Katsina et font la fierté de ses habitants. La plupart des témoignages sur birnin Katsina sont presque unanimes pour affirmer que « ses habitants sont plus pieux que tous les Hausawa et son souverain à elle (la ville de Katsina) est le plus pieux de tous les souverains hausa » comme nous le rapporte Hausawa da Makwabtansu en ces termes : "Mutanenta (Katsina) sun fi mutanen Hausa tsarki ; ita ta fi su sarki tsarkaka » 25 .

\section{La mosquée d'Agadez (Niger)}

L'histoire de l'Ayar en général et celle d'Agadez en particulier est jalonnée de mosquées qui témoignent de la vitalité et du dynamisme de l'islam dans cette zone ${ }^{26}$. La célèbre mosquée d'Agadez est située dans le quartier Katanga de Tsofon Gari. Elle porte le nom de mosquée d'Amiskine ${ }^{27}$. Le minaret qui la distingue de tous les bâtiments de la ville est haut de 27 mètres et semble avoir été construit, du moins sa première version, en $1515^{28}$. Ce minaret 
aurait eu une hauteur initiale de 29 mètres $^{29}$. En effet, selon l'étude de Cressier et Bernus, la mosquée aurait été construite en plusieurs étapes. Ainsi, après avoir passé en revue les différentes versions et interprétations sur la construction de cette mosquée, ces auteurs émettent les hypothèses suivantes $3^{30}$ :

"un premier édifice existe dès 1450 , et peut-être avant, suffisamment grand pour être mosquée de vendredi (si l'on croit les chroniques d'Agadez), mais sans doute sans minaret (ce qui justifierait le silence de J.-L. l'Africain). Plus tard, vers 1515 ou vers 1530 , selon la version retenue de la vie de Zakariya, ce monument initial aurait été reconstruit ou agrandi par celui-ci et le premier minaret aurait été élevé. Enfin, après sans doute d'autres modifications, qui n'ont pas laissé de trace dans la tradition au cours des trois siècles qui suivent, une reconstruction $d u$ minaret aurait eu lieu en 1844 ou $1847 » 31$.

La première mosquée sans minaret fut construite en 1449. Ensuite, vers 1500 ou 1515 , un minaret fut ajouté et, en 1847, des salles latérales. Cet audacieux bâtiment, construit en banco, a traversé les âges et reste l'édifice le plus élevé d'Agadez. Il fut un centre de formation qui accueillit des personnages intellectuels et religieux célèbres (Malam Jibril Dan Umaru, Usman Dan Fodio) et des leaders politiques comme Sir Ahmad Bello, le Premier Ministre
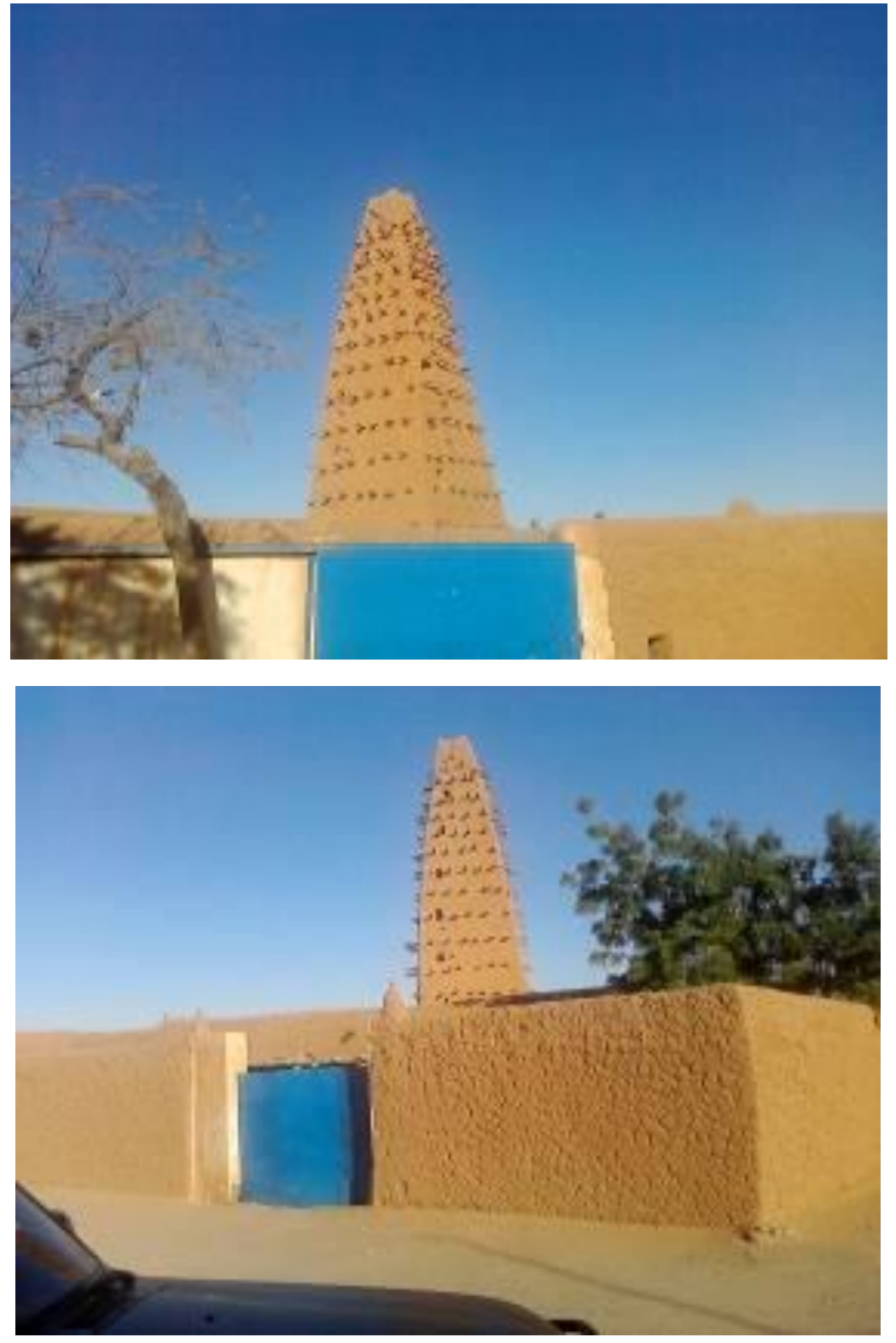

Figure 5: La mosquée d'Agadez, au Niger. Source: photographies de A. Mahamane, $1^{\text {er }}$ février 2013 
du Nord-Nigéria entre 1960 et 1966. Cette célébrité se maintient encore aujourd'hui avec l'utilisation du modèle de la mosquée d'Agadez pour en bâtir d'autres, comme en témoignent les photos ci-dessous, à Agadez même et à Arlit.

La mosquée Saudauna à Agadez, copie de la grande mosquée Amiskine (deux faces)

Cette mosquée encore en construction se situe dans la ville même d'Agadez. Elle rappelle la célèbre mosquée d'Amiskine par son minaret et ses salles laterals.

\section{Copie de la mosquée d'Agadez à Arlit} (Niger)

Ce minaret est hexagonal, celui d'Agadez est carré. Reconstituer l'histoire de la moquée d'Arlit nous permettra de souligner ses rapports avec les deux autres mosquées plus anciennes : la mosquée d'Amiskine et la mosquée de Tigida N’Tazamaq ou mosquée de Sardauna. Celle-ci, située dans la ville d'Agadez, fut reconstruite en 1964 par le Premier Ministre du Nord-Nigéria, Sir Ahmadu Bello, deux ans avant son assassinat le 15 janvier 1966.

\section{La mosquée de Bobo-Dioulasso (Burkina- Faso)}

La célèbre mosquée de Bobo-Dioulasso en banco, grand monument de couleur beige, a été construit par l'Almany Sidiki Sanou en 1880 et ressemble à une structure moderne ${ }^{2}$. Ses minarets hérissés de piquets ainsi que les contreforts de ses murs ont été modelés dans la latérite, et leurs bâtisseurs leur ont donné la forme d'obus ou de pains de sucre sur plan carré. À deux pas de la mosquée, se trouvent les
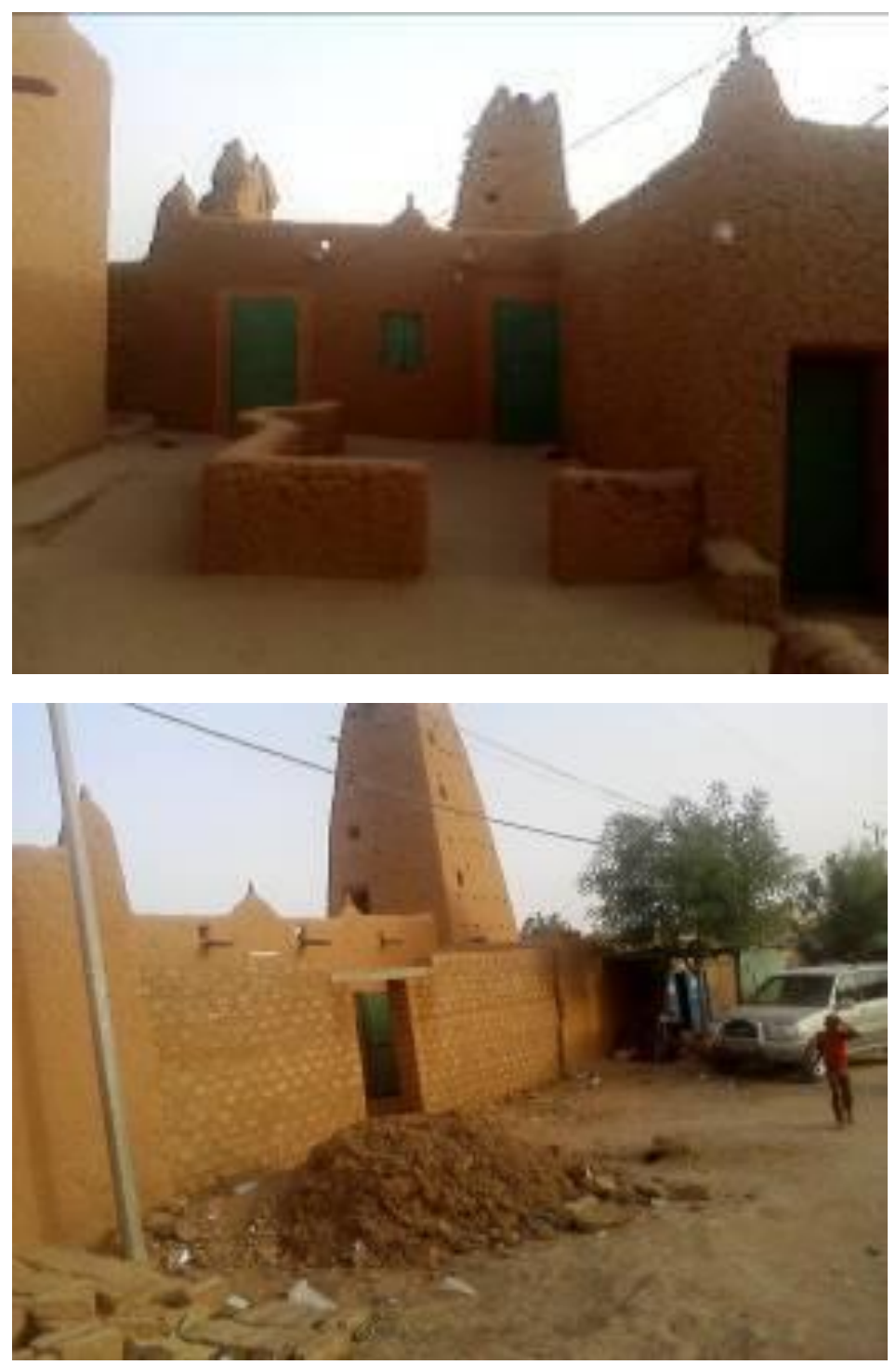

Figure 6: Mosquée Saudauna à Agadez, copie de la grande mosquée Amiskine (deux faces) à Agadez, au Niger. Source : photographies de A. Mahamane, 31 janvier 2013 


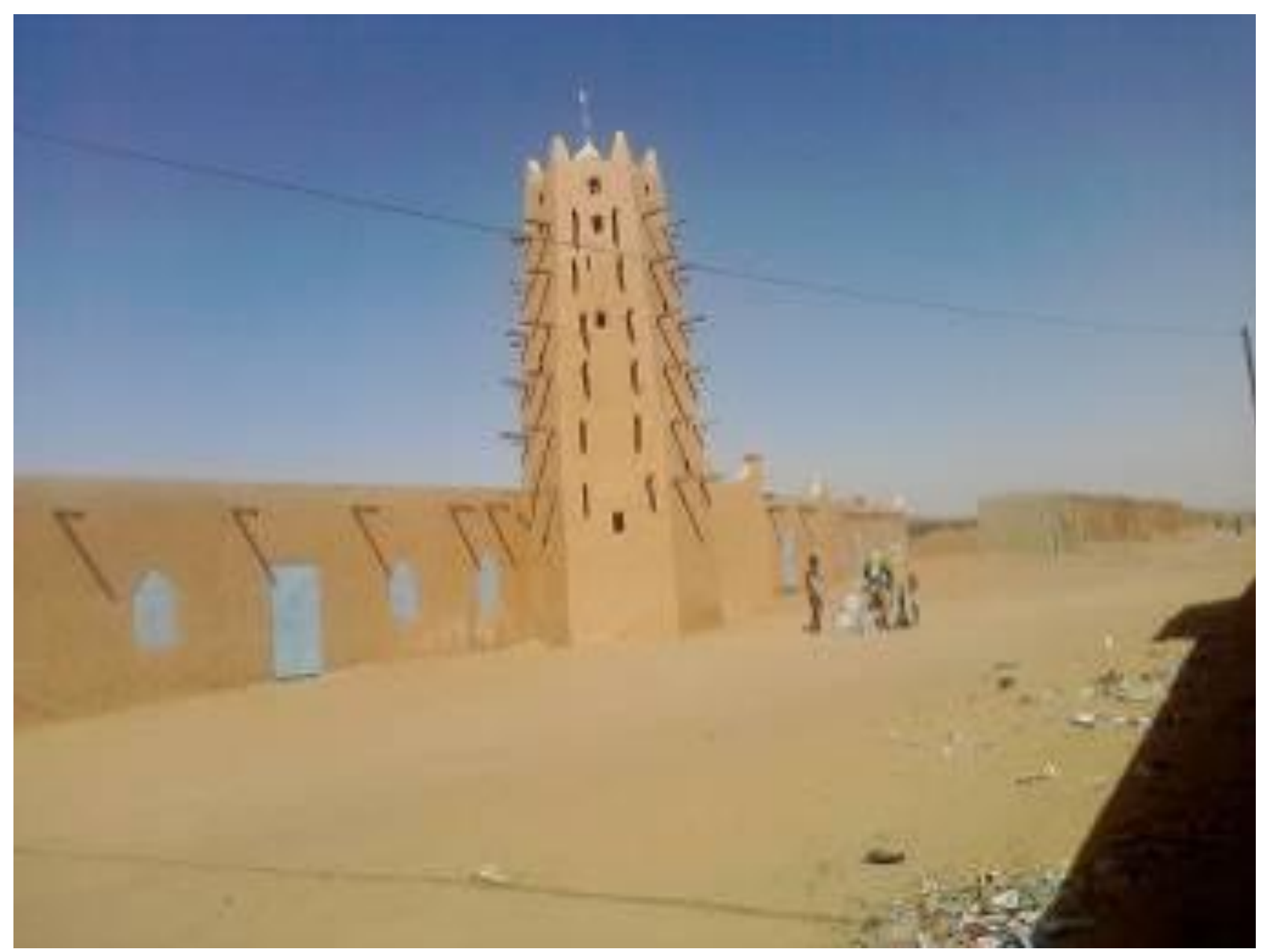

Figure 7 : Copie de la mosquée d'Agadez à Arlit, au Niger. Source : photographie de A. Mahamane, Arlit, 31 janvier 2013.

vieux quartiers de Kibidoué et de Sya, et leurs maisons en terre33. La mosquée de Bobo-Dioulasso rappelle à bien des égards les autres mosquées précoloniales d'Afrique de l'Ouest (du Mali, du Niger, du Nigeria). Nous reviendrons plus loin sur les caractéristiques, les symboles et les fonctions de ces mosquées précoloniales de l'Afrique de l'Ouest. La tour de la mosquée de Bobo-Dioulasso est une sorte de tour de contrôle du premier palais et de la capitale du pays de Daura. Ce premier palais est aujourd'hui un bloc rénové au sein du palais actuel. La sentinelle porte le titre de Bawan Dodo (le dévoué du monstre) prêt à affronter quiconque, y compris les esprits ou les génies, qui oseraient s'attaquer au souverain. 


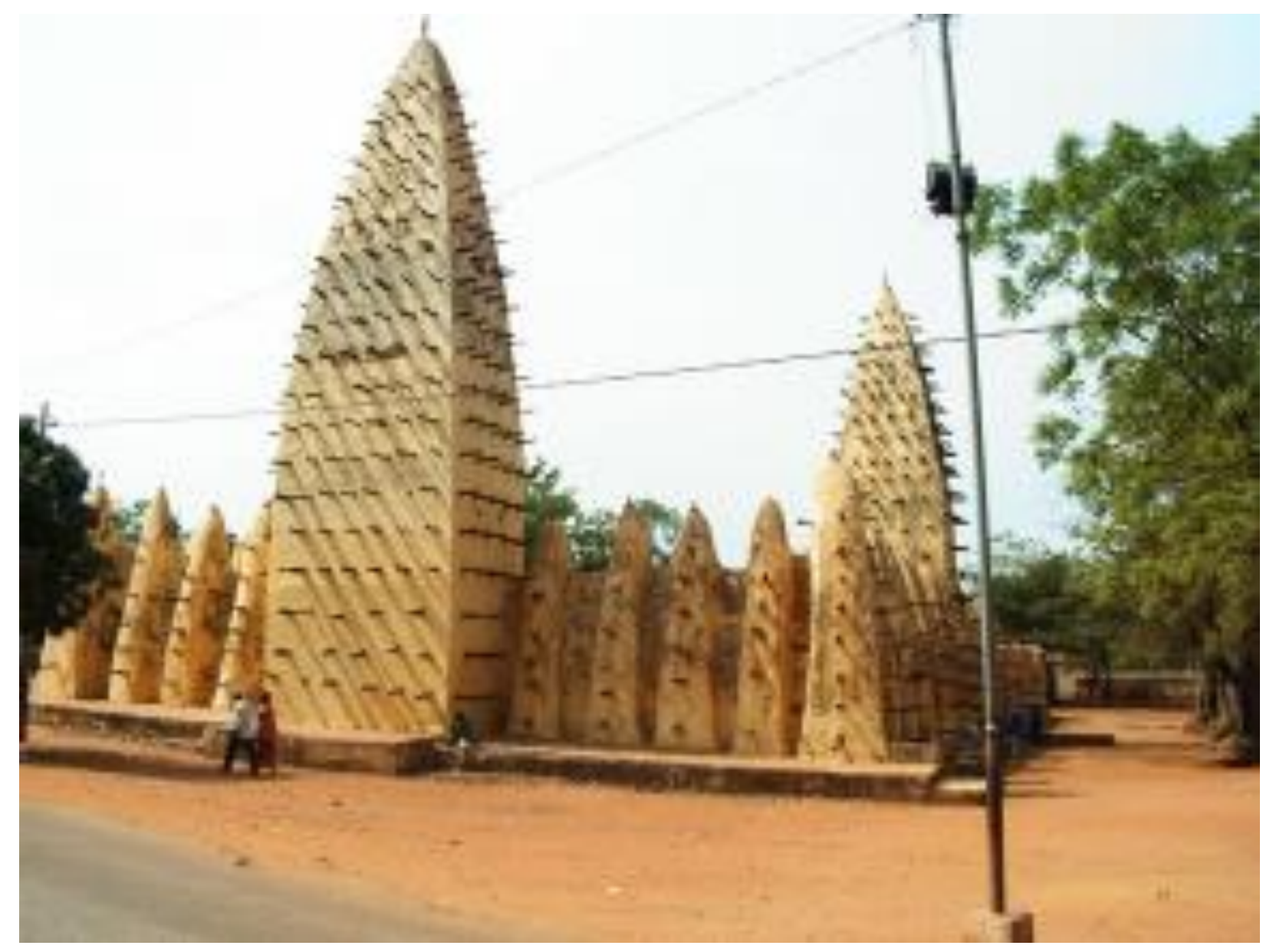

Figure 8 : La mosquée de Bobo-Dioulasso, au Burkina-Faso. Source : Wikipedia.org, le 16/o9/20o8

On ignore à quand remonte l'institution et la construction de cette tour. Elle remonterait à l'époque antéislamique ou aux époques de pratiques animistes et musulmanes simultanées. Son rôle a apparemment évolué dans le temps. Elle semble au départ être une tour dévouée aux pratiques mystiques et religieuses liées à la protection du souverain. La fonction de la tour s'est étendu par la suite au contrôle et à la protection du palais puis de toute la capitale de Daura et du royaume dans son ensemble. L'exemple de Soron Bawan Dodo nous montre que les anciennes capitales politiques précoloniales des États hausa avaient non seulement une protection physique par les murs d'enceinte (birni) ainsi que le ravin (garu) qui entourent la ville mais aussi une protection spirituelle par Bawan Dodo et humaine à travers l'armée et les administrateurs, notamment les 
responsables des portes de la ville et de la tour. Elle est, à notre connaissance, la seule tour du Soudan central qui n'était pas liée à l'islam. Elle est géographiquement très proche de la tour de Gobarau au Katsina, puisque les deux villes sont distantes de 77,94 kilomètres seulement. Ensuite, l'ancien État du Katsina et celui de Daura forment aujourd'hui un seul État de la République Fédérale du Nigéria, avec comme capitale Birnin Katsina.

\section{Caractéristiques principales des monuments analyses}

\section{La question de la chronologie des monuments étudiés}

La première question, relative à ces monuments, qui se pose à l'historien est celle de la chronologie. L'établissement de cette chronologie suppose un recensement exhaustif de tous les monuments historiques du Sahel afin de disposer non seulement d'un tableau du patrimoine de la zone dans le domaine étudié mais aussi d'inscrire ce patrimoine dans le temps et l'espace. Ce recensement et cette question de chronologie ouvrent un vaste chantier de recherche sur le patrimoine matériel et immatériel du Sahel. L'échantillon examiné ici ne concerne que quatre pays : le Burkina Faso, le Mali, le Niger et le Nigéria, mais un travail d'ensemble sur la question serait nécessaire. La classification chronologique des échantillons signalés dans cette étude pose de sérieux problèmes, qui sont de plusieurs ordres. D'abord, nous ne disposons pas de dates repères absolues dans chacun des cas étudiés. Ensuite, la tradition orale de chacun est liée à des légendes, des mythes et des confusions tendant à faire de chaque cas le plus ancien. Mais les difficultés de classification chronologique des échantillons sont également liées au transfert des sites, aux reconstructions, rénovations et aux reproductions souvent très difficiles à situer dans le temps. La durée de la construction des ouvrages n'est pas connue dans la mesure où on ne connaît pas les dates précises du début de construction, de finition et de la première date de mise en service de chaque ouvrage.

Certains monuments sont donc, comme Soron Bawan Dodo, chronologiquement inclassables. Car on ne sait pas si le premier soro a été construit dans l'ancienne capitale de Daura ou si le monument actuel du deuxième site de Daura, dit Birnin daura, est une innovation ou une réplique d'une pratique préexistante. C'est aussi le cas de la mosquée d'Agadez, dont les spécialistes les plus pointus placent le début de la construction à 1450 et sa reconstruction ou son agrandissement entre 1515 et 1530, alors que l'année de sa construction la plus communément retenue est 1515 . Si les hypothèses les plus répandues font remonter la construction de la Grande mosquée de Djenné au XIII e siècle, la construction ou la reconstruction du bâtiment

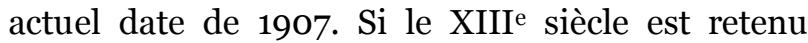
comme le moment approximatif de la construction de cette mosquée, elle serait la doyenne de toutes les mosquées évoquées dans cet article.

La construction de la mosquée de Djingareyber aurait débuté vers 1325 et terminée en 1328 sous la supervision technique de l'architecte andalou Abou Ishaq es-Sahéli34. Celle-ci, du moins le premier bâtiment, serait postérieure à celle de Djenné. Viendrait ensuite celle de Sidi Yaya qui aurait été érigée vers 1440 . Si l'on attribue la construction de la mosquée de Gobarau à Muhammad Korau (14451493), elle serait la « petite sœur » de la mosquée de Sidi Yaya. Mais si on situe sa construction à l'époque des saints Dan Marina décédé vers 1529 et Dan Masani né en 1595, elle serait postérieure à la mosquée d'Agadez dont la date indicative du début de la construction est 1515. Selon la chronologie d'Usman, Dan Marina est bien contemporain de Dan Masani et est décédé vers $1655^{35}$. La mosquée de Bobo Dioulasso, construite par l'Almany Sidiki Sanou en 1880, serait la benjamine de tous les immeubles de la période précoloniale examinés dans la zone étudiée. 


\section{Formes et fonctions des monuments}

Des salles collatérales sont dominées par des minarets en forme de pyramide, d'obus, de pain de sucre, qui piquent vers le ciel. Ces minarets (Hasumiyar Gobarau, Amiskine, Gingareyber, etc.) ou étage supérieur du serviteur du monstre (Soron Bawan Dodo) représentent une sorte de tour à deux fonctions au moins. Ils sont d'abord des minarets pour l'appel à la prière musulmane, à l'exception du Soron Bawan Dodo. Dans cette fonction, ils sont utilisés au moins cinq fois par jour. Ils constituent aussi une sorte de "tour de contrôle" et de surveillance du palais, de la ville ${ }^{36}$ ou du territoire. Selon Hamani, la mosquée d'Agadez porte encore aujourd'hui le nom d'Amiskine. Ce terme signifierait en tamasheq «ce qui permet d'apercevoir de loin »37. Dans ces deux fonctions appel à la prière et surveillance - les mosquées, les minarets et Soron Bawan Dodo sont directement associés au pouvoir politique et à la gouvernance de l'époque.

L'histoire de tous ces bâtiments est liée à des mythes et légendes, mais également au progrès de l'islam dans la région. Ceux-ci sont donc les dépositaires de certaines croyances et valeurs religieuses et spirituelles. En effet, ces bâtiments constituaient et constituent encore des lieux d'étude et de formation religieuse, politique et sociale. Certains d'entre eux, sinon tous, étaient érigés en véritables universités réputées pour leurs sciences islamique, politique, sociale et technique.

Tous les bâtiments furent construits avec des matériaux locaux : piquets, contreforts, banco, latérite, eau, etc. Ce qui frappe ici, c'est le caractère durable de ces matériaux d'usage traditionnel adaptés à l'environnement, avec leurs déclinaisons techniques et esthétiques, marqueurs culturels et vecteurs du patrimoine immatériel ${ }^{8}{ }^{8}$. La pérennité des bâtiments témoigne de l'existence d'un patrimoine technologique et d'un savoir-faire dépositaires du patrimoine immatériel soudano- sahélien, axé sur la spiritualité, l'enseignement, les outils de légitimation et de sécurisation du pouvoir.

\section{Conclusion}

Nous sommes ici, dans le cas d'un patrimoine à plusieurs dimensions où le matériel, le spirituel, le politique et le technique sont intimement liés. Des travaux d'ensemble sur cette question sont indispensables pour élargir et approfondir nos connaissances sur ce patrimoine et redécouvrir des savoir-faire architecturaux traditionnels. Tous ces bâtiments constituent également des véritables lieux de mémoire et d'acquisition du savoir religieux. Les recherches sur ces bâtiments, leurs matériaux et les techniques de leur construction pourraient contribuer à résoudre certains problèmes de développement notamment l'importation à grand coût de matériaux de construction produits hors des zones qui abritent les bâtiments. En effet, les techniques de construction traditionnelles, leur collecte, leur conservation, leur étude et leur connaissance, pourraient servir de base à la recherche d'un développement durable en matière de construction grâce aux matériaux endogènes ou sous-régionaux.

1 Cet article s'inscrit dans le cadre d'un programme de recherche sur les palais royaux, les mosquées et la gouvernance au central Soudan du XVe au XIXe siècle.

2 Les palais royaux du Katsina, le Palais du sultan du Damagram, etc.

3 La mosquée ou tour de Gobarau au Katsina, Nigéria (Mahamane 1998), la mosquée d'Agadez au Niger (Cressier et Bernus 1984), la mosquée d'Ahmadu Sékou à Hamdallayi (Mopti), les mosquées de Sankore et Gingarey Beri (La Grande Mosquée, en zarma) à Tombouctou, la mosquée de Djenné, etc. (Ki-Zerbo, 1978)

4 Soron Bawan Dodo dans le palais royal de Daura (Nigéria). Soron Bawan Dodo (Moussa Ibrah 2008) signifie en hausa «l'immeuble du serviteur du monstre ». 
5 C'est le cas de la ville d'Agadez, le palais du Sultan et la célèbre mosquée de la ville, entre autres, classée en 2013 patrimoine de l'Humanité.

\section{KI-ZERBO, 1978 : V, VII-XIII, XVIII-XXIII, 186-188).}

7 Les ruines de Loropéni au Burkina Faso sont bardées de hauts murs et s'étendent sur $11130 \mathrm{~m}^{2}$. C'est la mieux préservée des dix forteresses que compte la région du Lobi. Vieilles d'au moins mille ans selon des découvertes récentes, ces ruines sont situées près des frontières du Togo et du Ghana. http://www.linternaute.com/voyage/magazine/photo/patrimoin e-mondial-de-l-unesco-les-nouveaux-inscrits/les-ruines-deloropeni-au-burkina-faso.shtml (du o9 juillet 2014).

Une brochure du Ministère de la Culture, du Tourisme et de la Communication du Burkina Faso, intitulée les ruines de Loropéni éditée en juillet 2009, donne assez de détails et de précisions sur l'architecture de ce site. «Dans le Sud-ouest du Burkina Faso, dans la Province de Poni en particulier, se trouvent des ruines de constructions anciennes en pierres, signalées dans la littérature déjà au début du XXe siècle. Ces constructions qu'on dénombre par centaines, débordent les frontières du Burkina Faso, puisqu'on les retrouve aussi en Côte d'Ivoire. D'après leur morphologie, on peut les classer en deux grands groupes: certaines enceintes sont quadrangulaires et d'autres circulaires. Elles sont toutes bâties en moellons de latérites liés par un mortier d'argile gravillonneuse. Les ruines quadrangulaires sont parmi les plus grandes. Elles peuvent atteindre 100 mètres de côté comme à Loropéni. La forme circulaire concerne des cercles ou des petites enceintes mesurant 8 à $15 \mathrm{~m}$ de diamètre » (MCTC $2009: 2$ ).

8 C'est le cas au Niger en 2012 où une école s'est écroulée, occasionnant mort d'homme dans la région de Tillabery. Un autre cas s'est aussi produit, toujours au Niger, en juillet 2014 à Niamey, occasionnant la mort de deux personnes. Malheureusement ces exemples ne sont pas des exceptions dans la sous-région.

9 Mansa Moussa ou Kankou Moussa (1312-1332) entreprit son célèbre pèlerinage en 1324 (Ki-Zerbo $1978: 135$ ).

10 CISSOKO $1966: 55$ et Ki-Zerbo $1978: 136$.

11 Un poète architecte, originaire de Grenade, Abou Ishaq, dit Es Saheli selon Ki-Zerbo (1978: 136).

12 CISSOKO $1966: 55$.

13 http://fr.wikipedia.org/wiki/Mosqu\%C3\%Age Djingareyber, (consulté le 26/10/2013).

14 Le banco ici signifie de l'argile. Dans l'architecture sousrégionale, cette argile subit souvent plusieurs semaines ou mois de préparation minutieuse avant d'être utilisée dans la construction. Dans certains cas la latérite est utilisée à la place de l'argile dans les mêmes conditions de préparation.

15 1325: L'empereur Mansa Moussa débute la construction d'une Mosquée prestigieuse. 1328 : Achèvement de la mosquée de Djingareyber. 1353: Le voyageur Tangérois Ibn Battûta visite la ville. XVe siècle : Construction de la mosquée de Sankoré, aux dimensions de la Kaaba (http://yacinfo.over-blog.net/articlereseaux-en-classe-introduction-au-reseau--38260700.html (consulté le 26 octobre 2013).

16 http://fr.wikipedia.org/wiki/Mosqu\%C3\%A9e_Djingareyber (consulté le 26/10/2013).

17 Cette présentation de la grande mosquée de Djenné a été faite à partir du site suivant : http://fr.wikipedia.org/wiki/Grande_mosqu\%C3\%Age_de_Dje nn\%C3\%A9 (consulté le 26 octobre 2013).

18 Gobarau est le nom du quartier qui abrite cette tour au sujet de laquelle il n'existe qu'une seule source : un texte en anglais et en hausa, qui peut être obtenu auprès de Malam Sani Dan Baushi, "Katsina State History and Culture Bureau », department of Documentations and Research, Dossier $n^{\circ} 101$. Le texte en hausa fait 1 p. et celui en anglais, 2 p. Sani Dan Baush témoigne avoir recueilli ce texte en janvier 1989 (entretien individuel enregistré à Katsina le 30 décembre 1996).

19 Mahamane, $1998: 384$ et note 23 et aussi les pages 381-383 sur la question. Les témoignages écrits les plus anciens sur le Katsina ne parlent pas de cette tour alors qu'il semble certain qu'elle ait été construite avant le XIX ${ }^{e}$ siècle, dans la mesure où aucun émir issu du jihad ne revendique sa réalisation. Nous retrouvons d'ailleurs la même situation avec la légende de Bayajida qui n'est rapportée que par les documents écrits du $\mathrm{XIX}^{\mathrm{e}}$ siècle, alors que ceux connus antérieurement au XIX ${ }^{\mathrm{e}}$ siècle ne font pas référence à cette légende.

20 Voir par exemple cette tradition : Un mythe populaire sur l'origine de Gobarau stipule que lorsque Muhammad Korau tua Jibda-Yaki Sanau, le dernier roi païen de Katsina, il voulut construire une mosquée. Une fois le site choisi, il y eut le problème de la direction de la Qibla (orientation de la prière vers le Ka'abah de La Mecque à laquelle la mosquée doit faire face). Muhammad Korau consulta les savants musulmans de l'époque, et ils acceptèrent, sauf un saint du nom de Mallam Jodoma, qui était un inconnu. Une dispute éclata, les autres savants insultèrent Jodoma et le traitèrent d'étranger qui voulait amener l'instabilité dans le pays. Mallam Jodoma se mit en colère, pointa son doigt vers une autre direction qui se révéla être la direction recherchée de la Ka'aba. Muhammad Korau, surpris, nomma Mallam Jodoma Imam principal du souverain, au grand dam des autres imams, qui firent croire à Muhammad Korau que Jodoma, 
devenant de plus en plus célèbre, voulait son trône. Jodoma fut alors banni de Katsina, et il s'installa à Guga, un village aujourd'hui du nom de Bakori, où il mourut (http://en.wikipedia.org/wiki/Gobarau_Minaret (consulté le 26 octobre 2013).

21 http://en.wikipedia.org/wiki/Gobarau_Minaret (consulté le 26 octobre 2013).

22 HAUSAWA DA MAKWATANSU, I, Zaria, N.N.P.C., 1970, p. 9. MALLAM UBAN DOMA S. S. M. Dan et BOYD Jean, Infakul maisuri na sarkin musulmi Muhammadu Bello. Fasara a takaice. Zaria, N.N.P.C., 1974, p. 82. USMAN B. Y., "The birane of Katsina ", Cities of the Savannah, Lagos, Nigeria Magazine Publications, 1979, pp. 40-41.

23 KITAB MASA-ALAT AHBAR KATSINA (KIMAKA), "Livre sur les nouvelles du Katsina» by B. Ulamai. Nigerian National Archives Kaduna/P/AR11/1. Le "KATSINA », Nigeria Magazine, $1956, \mathrm{n}^{\circ} 51$, p. 308, rapporte que la tour de Gobarau date du XVII ${ }^{\text {e }}$ siècle. Alors que la tradition enseigne que sa construction remonte au $\mathrm{XV}^{\mathrm{e}}$ siècle - sur la tradition orale, voir supra.

24 USMAN Y. B., op. cit., 1981, PP. 84, 94. Les témoignages écrits les plus anciens sur le Katsina ne parlent pas de cette tour alors qu'il semble certains qu'elle soit construite avant le XIX ${ }^{\mathrm{e}}$ siècle dans la mesure où aucun émir issu du jihad ne revendique sa réalisation. Nous retrouvons d'ailleurs la même situation avec la légende de Bayajida qui n'est rapportée que par les documents écrits du XIX ${ }^{e}$ siècle alors que ceux connus antérieurement au $\mathrm{XIX}^{\mathrm{e}}$ siècle ne font pas mention de cette légende.

25 HAUSAWA DA MAKWATANSU, I, op. cit., 1979, p. 9. "Ses (Katsina) populations sont plus pieuses que toutes les populations hausa. Leur roi est le plus pieux de tous les Etats Hausa. ». Nous avons traduit tsarki, ici, par pieux. Généralement il se traduit par saint. Mais la notion de saint ne convient pas à ce contexte. PUBLIC RECORD OFFICE, C.O.465, Northern Nigeria, $\mathrm{n}^{\circ} 3,1902, \mathrm{p}$. $\mathrm{U}$ : "Katsina in the Northern is the great centre of Mohammenden education". "KATSINA », Nigeria Magazine, $1956, \mathrm{n}^{\circ} 51$, pp. 310, 315

26 CRESSIER et BERNUS, 1984: 5-43, HAMANI, 1989: 156157, 195-198 et ABDOU, 201: 53-662. Ce constat est confirmé par nos observations sur le terrain en janvier et février 2013.

27 Amiskine signifierait en Tamasheq (langue des Touareg) : «ce qui permet d'apercevoir de loin » (Hamani 1989: 157).

28 http://www.agadez-niger.com/page-agadezmosquee.htmlhttp://www.agadez-niger.com/page-agadezmosquee.html (consulté le 26 octobre 2013).
29 ABADIE, 1927 : 199.

30 CRESSIER et BERNUS, $1984: 5$-9.

31 CRESSIER et BERNUS, $1984: 8$.

32 C'est-à-dire à un monument conçu et réalisé par les architectes et les techniciens formés dans les écoles spécialisées de l'époque contemporaine.

33 http://www.cultureetpartage.com/index/article/id/919/lamosquee-dioulassoba-bobo-dioulasso-au-burkina-faso (consulté le 26 octobre $2013-10-26$ )

34 KI-ZERBO, $1978: 135$.

35 MAHAMANE, $1998: 151$ et 112 note 26

36 Hasumiya est un terme hausa emprunté à l'arabe qui signifie tour. Selon les traditions du Katsina, la tour de Gobarau était aussi utilisée pour le contrôle de l'arrivée des caravanes ou des éventuels attaquants.

37 HAMANI, 1989: 157: La ville d'Agades vit au rythme des échanges et de transactions. L'arrivée des caravanes est donc un événement extrêmement important et une occasion de ravitaillements, d'affaires et de réjouissances.

38 Cet aspect sera décrit et commenté dans nos travaux ultérieurs. 


\section{Bibliographie}

ABADIE, Maurice, La colonie du Niger, Paris, Sociétés d'Éditions Géographiques, Maritimes et Coloniales, 1927.

ABDOU, Mounkaila, Répertoire des sites historiques et des lieux de cultes musulmans dans la ville d'Agades, Mémoire de Maitrise d'Histoire, Université de Niamey, Faculté des Lettres et Sciences Humaines, année académique 2012.

ADAMOU, Aboubacar, Agadès et sa région. Contribution à l'étude du Sahel et du Sahara nigériens, Niamey, I.R.S.H., 1979.

CRESSIER Patrice et BERNUS Suzanne, «La grande mosquée d'Agadez: Architecture et histoire », Journal de la Société des Africanistes, Tome 54, Fascicule 1, 1984, pp. 5-43.

CISSOKO, CISSOKO, S.-Mody, Histoire de l'Afrique occidentale. Moyen-âge et Temps modernes, VII e siècle-1850, Paris, Présence Africaine, 1966, 336 p.

HAMANI, Djibo, Au carrefour du Soudan et de la Berbérie. Le sultanat touareg de l'Ayar. Niamey, IRSH, 1989.

KI-ZERBO, Joseph, Histoire générale de l'Afrique. D'Hier à Demain, Paris, Hâtier, 1978, $736+$ XXXI p.

MAHAMANE, Addo, "Les relations entre le monde hausa et le monde songhay » Mu Kara Sani, 10 (1), 2002, pp. 57-74.

MAHAMANE, Addo, Institutions et imaginaires politiques hausa : le cas du Katsina sous la dynastie de Korau (XVe-XIXe siècles), thèse de doctorat unique. Aix-en-Provence, France, 1998.

MINISTERE DE LA CULTURE, DU TOURISME ET DE LA COMMUNICATION (MCTC), Les ruines de Loropéni, Ouagadougou, MCTC, Juillet 2009, $16 \mathrm{p}$.

\section{Webographie}

http://yacinfo.over-blog.net/article-reseaux-enclasse-introduction-au-reseau--38260700.html (du 26 octobre 2013).

http://fr.wikipedia.org/wiki/Mosqu\%C3\%A9e_ Djingareyber, (du 26/10/2013).

http://yacinfo.over-blog.net/article-reseaux-enclasse-introduction-au-reseau--3826070o.html, (du 26 octobre 2013).

http://fr.wikipedia.org/wiki/Grande_mosqu\%C 3\%A9e_de_Djenn\%C3\%A9, (du 26 octobre 2013).

http://en.wikipedia.org/wiki/Gobarau_Minaret, (du 26 octobre 2013).

http://www.cultureetpartage.com/index/article /id/919/la-mosquee-dioulassoba-bobo-dioulassoau-burkina-faso, (du 26 octobre 2013-10-26).

http://www.agadez-niger.com/page-agadezmosquee.htmlhttp://www.agadez-niger.com/pageagadez-mosquee.html, (du 26 octobre 2013). 